\title{
Arrhythmia Detection based on Morphological and Time-frequency Features of T-wave in Electrocardiogram
}

\author{
Elham Zeraatkar, Saeed Kermani, Alireza Mehridehnavi ${ }^{1}$, A. Aminzadeh ${ }^{2}$, E. Zeraatkar ${ }^{3}$, Hamid Sanei ${ }^{4}$ \\ Departments of Physics \& Biomedical Engineering and ${ }^{4}$ Internal Medicine, ${ }^{1}$ Medical Image E Signal Processing Research Center, \\ Isfahan University of Medical Sciences, Isfahan, ${ }^{2}$ Navigation and Control, Marine Industries Organization, ${ }^{3}$ Electronic and Computer \\ Engineering, Shiraz University, Shiraz, Iran
}

\section{A B S T R A C T}

As the T-wave section in electrocardiogram (ECG) illustrates the repolarization phase of heart activity, the information which is accumulated in this section is so significant that it can explain the proper operation of electrical activities in heart. Long QT syndrome (LQT) and T-Wave Alternans (TWA) have imperceptible effects on time and amplitude of T-wave interval. Therefore, T-wave shapes of these diseases are similar to normal beats. Consequently, several T-wave features can be used to classify LQT and TWA diseases from normal ECGs. Totally, 22 features including 17 morphological and 5 wavelet features have been extracted from T-wave to show the ability of this section to recognize the normal and abnormal records. This recognition can be implemented by pre-processing, T-wave feature extraction and artificial neural network (ANN) classifier using Multi Layer Perceptron (MLP). The ECG signals obtained from 142 patients (40 normal, 47 LQT and 55 TWA) are processed and classified from MIT-BIH database. The specificity factor for normal, LQT, and TWA classifications are $99.89 \%, 99.90 \%$, and $99.43 \%$, respectively. T-wave features are one of the most important descriptors for LQT syndrome, Normal and TWA of ECG classification. The morphological features of T-wave have also more effect on the classification performance in LQT, TWA and normal samples compared with the wavelet features.

Key words: ECG, feature extraction, morphology, neural network, T-wave, wavelet

\section{INTRODUCTION}

The long QT syndrome (LQTs), T-wave alternans (TWA), and ventricular tachyarrhythmia (VT) are some of the common cardiac diseases which cause sudden cardiac death $(\mathrm{SCD})$ in the world. ${ }^{[1,2]}$ Many studies have been developed to detect an abnormal sinus ECG based on the features of ECG signal. Most of these articles use QRS complex to indentify the arrhythmia of the heart. One of the traditional methods has been performed by Jain ${ }^{[3]}$ that digitized and represented each ECG lead by its z-domain modes to enhance the discrimination of the subtle changes in $\mathrm{P}, \mathrm{QRS}$, and $\mathrm{T}$ sections, the derivatives of the waves are employed for extraction of the modes. Lin et al. ${ }^{[4]}$ used linear prediction to extract features from QRS complexes. Osowski et al. ${ }^{[5]}$ applied fuzzy neural network to ECG beat recognition and classification and the features drawn from the higher order statistics have been proposed in the study. Also Engin ${ }^{[6]}$ performed similar method and used autoregressive model coefficients, higher-order cumulant, and wavelet transform variances as features to enhance the performance. Jekova et al ${ }^{[7]}$ implemented four different classifiers based on 26 morphological features which have been extracted from lead I, II, and the Frank Leads or vector cardiograph (VCG) trajectory signals, such as area, slopes, peaks, time intervals, and VCG diagram in QRS complex. Asl et al. ${ }^{[8]}$ presented an effective cardiac arrhythmia classification algorithm based on the generalized discriminant analysis (GDA) to reduce feature scheme using support vector machine (SVM) classifier. Initially, 15 different linear and nonlinear features have been extracted from QRS complex and then reduced to only 5 features by the GDA technique. Vaglio et al. ${ }^{[9]}$ and Couderc et al. ${ }^{[10]}$ implemented a computer algorithm to identify the differentiation of LQT1 and LQT2 carriers' base on $\mathrm{T}$-wave morphology features, such as the $\mathrm{Q}$ to T-peak (QT-peak), the T-peak to T-end interval, T-wave magnitude, and T-loop slopes in these studies.

In recent works, ${ }^{[11,12]}$ simulated and synthetic TWA signals were generated. These augmented beats were detected using wavelets and $91 \%$ sensitivity was achieved. In other studies, wavelet/FFT ${ }^{[13]}$ and correlation/FFT methods ${ }^{[14]}$ were also considered.

The novelty of this work can be explained as follows. In 
this study, diseases are recognized from the beats that seem normal (sinus ECG), but in fact, they belong to LQT or TWA classes. However, other works only classify heartbeat types. ${ }^{[11-14]}$ Consequently, previous studies are not comparable with this approach.

In this article, diseases are classified by the following procedures: Pre-processing, QRS-complex detection, T-wave detection, features extraction from T-wave section (morphological and wavelet coefficients), and classification using MLP artificial neural networks.

\section{METHOD}

Arrhythmia detection algorithm is implemented as follows: (a) recalling suitable ECG database; (b) pre-processing; (c) QRS-complex detection; T-wave detection; (d) feature extraction from T-wave; and (e) MLP classifier as shown in Figure 1.

\section{ECG Database and Pre-processing}

In this article, the MIT/BIH database ${ }^{[15-17]}$ has been chosen with 40 normal records, 47 LQT syndrome records, and 55 sets of TWA arrhythmia from 142 ECG recordings Lead I with $128 \mathrm{~Hz}, 250 \mathrm{~Hz}$, and $500 \mathrm{~Hz}$ sampling rate, respectively.

Before applying detections, feature extraction and classifying procedure in this experiment, several preprocessings are necessary to obtain an appropriate result and reduce errors in processing and detection phases. Most common artifacts and drifts appear by $50 \mathrm{~Hz}$ of $60 \mathrm{~Hz}$ power line interface, muscle contractions of electromyography noise (EMG), baseline drift and ECG amplitude modulation with respiration, and ECG corruption with abrupt baseline shift. ${ }^{[18]}$ To avoid these disturbances, the following filters are applied:

a. To eliminate power line effect a notch filter ${ }^{[19]}$ has been developed with the following transfer function:

$$
H(z)=\frac{\left(z-z_{1}\right)\left(z-z_{2}\right)}{\left(z-p_{1}\right)\left(z-p_{2}\right)}
$$

Where

$$
z_{1}=\cos \left(\omega_{0}\right)+j \sin \left(\omega_{0}\right)
$$

And

$$
z_{2}=\cos \left(\omega_{0}\right)-j \sin \left(\omega_{0}\right)
$$

are the zeros of transfer function and

$$
p_{1}=k\left[\cos \left(\omega_{0}\right)+j \sin \left(\omega_{0}\right)\right]
$$

And
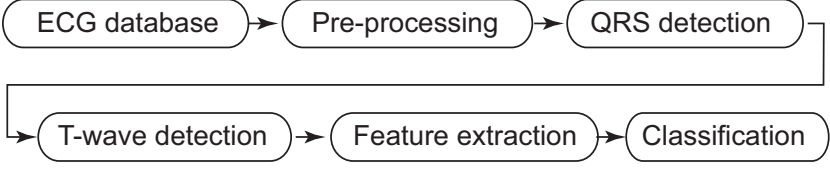

Figure I: Block diagram of algorithm

$$
p_{2}=k\left[\cos \left(\omega_{0}\right)-j \sin \left(\omega_{0}\right)\right]
$$

are the poles of the transfer function with pole/zero ratio $k=0.9$, cutoff frequency $\omega_{0}= \pm\left[f_{0} / f_{s}\right] \times(2 \pi \mathrm{r})$, center frequency $f_{0}=50 \mathrm{~Hz}$ and sampling rate $f_{s}$.

b. To reduce the effect of EMG noise, a discrete Butterworth filter with order 8 and cutoff frequency $f_{c}=70 \mathrm{~Hz}$ and sampling rate $f_{s}$.

c. For decreasing the amount of ECG baseline drift with respiration the following method is applied: ${ }^{[20]}$

1. Computing median of the ECG

2. Shifting ECG by this median value

3. Fitting a $4^{\text {th }}$ degree polynomial to the shifted ECG

4. Shifting ECG by this calculated polynomial

5. Detecting the R peaks of ECG

6. Computing median of each RR interval the ECG

7. Shifting each RR interval by its median value

d. For ECG corruption with abrupt baseline shift the algorithm mentioned in part(c) is also applied to the ECG signal.

Noise reduction and robustness of implemented algorithm with above artifacts have been discussed in another study. ${ }^{\mid 21]}$

\section{QRS Complex Detection}

In this section, the QRS complexes of the ECG are detected and eliminated form overall ECG to prepare the signal for $\mathrm{T}$-wave detection. This will be implemented by the following steps: ${ }^{[22]}$

1. Recall ECG signal $S(n)$ and compute square of this signal after pre-processing:

$T S 1(n)=S(n) * S(n)$

2. Evaluate the steepest windowed gradient of TS1(n) by using a rectangular sliding window with 11 points from sample $n-5$ to $n+5$ :

$G 1(n)=T S 1_{\text {max }}(w)-T S 1_{\text {min }}(w)$

3. Smooth the signal by using a moving average method from sample $n-5$ to $n+5$ with center $n$ :

$F G 1(n)=\frac{1}{11} \sum_{i=n-5}^{n+5} G 1(i)$ 
4. Normalize the following values by their respective maximum peak amplitude: $S(n), T S 1(n), G 1(n)$ and FG1(n).

5. Transform the ECG signal by a sigmoid function:

$$
\operatorname{TS} 2(n)=1-\frac{2}{e^{2 S(n)}+1}
$$

6. Evaluate the steepest windowed gradient of TS2(n) by using a rectangular sliding window with 11 points from sample $n-5$ to $n+5$.

$G 2(n)=T S 2_{\max }(w)-T S 2_{\min }(w)$

and smooth it to $F G 2(n)$ like step 3.

7. Normalize the following values by their respective maximum peak amplitude: TS2(n), G2(n) and FG2(n).

8. Multiply by ECG with $F G 2(n)$ :

$T S 3(n)=F G 2(n) * S(n)$

9. Evaluate the steepest windowed gradient of TS3(n) by using a rectangular sliding window with 11 points from sample $n-5$ to $n+5$.

$G 3(n)=T S 3_{\text {max }}(w)-T S 3_{\min }(w)$

and smooth it to FG3(n).

10. Normalize the following values by their respective maximum peak amplitude: TS3(n), G3(n) and $F G 3(n)$.

11. Compute:

$T S 4(n)=F G 1(n)+F G 3(n)$

12. Shift the resulting signal by median ' $m$ ':

$T S 4 m(n)=T S 4(n)-m$

13. Normalize $\operatorname{TS} 4 m(n)$ as:

Pre_F $F_{Q}(n)=T S 4 m(n) / \max (a b s(T S 4 m(n)))$

14. $F_{0}(n)$ is derived by retaining the amplitude values of Pre_FQ exceeding $5 \%$ of its maximum peak amplitude and reducing the remaining to zero:

$$
F_{Q}(n)=\left[\begin{array}{cr}
\operatorname{Pr} e_{-} F_{Q}(n) \text { if } \operatorname{Pr} e_{-} F_{Q}(n)>0.05 \\
0 & \text { otherwise }
\end{array}\right.
$$

15. $C_{0}$ is the proposed feature signal employed for identifying QRS out of ECG signal. This signal is digitalized version of $F_{Q}(n)$ :

$$
C_{Q}(n)=\left[\begin{array}{cc}
1, & \text { if } F_{Q}(n)>0.05 \\
0, & \text { otherwise }
\end{array}\right]
$$

\section{T-Wave Detection}

To extract features from T-wave section of ECG signal the interval of T-wave segments should be separated from other parts of signal. There are several methods to detect this section. ${ }^{[23-26]}$ One of the latest approaches can be done by the following steps after eliminating negative values and QRS parts of ECG signal in each of the following steps:

1. $f c 1$ : Feature \#1 is calculated from the first derivative of ECG signal

2. $f c 2$ : Feature \#2 is calculated from filtered gradient, which means the ECG signal passes through a sigmoid function, windowed gradient, and smoothed by a moving average window

3. $f c 3$ : Feature \#3 is calculated from the product of filtered gradient ECG and $f c 2$

4. $f c 4$ : Feature \#4 is calculated from the combination of $f c 1, f c 2, f c 3$ and absolute value of ECG: $[f c 1+f c 2+f c 3+\mid S(n)]^{*}|S(n)|$

5. fc5: Feature $\# 5$ is calculated from another combination of $f c 1, f c 2, f c 3$ and absolute value of ECG: $f c 1+f c 2+f c 3+|S(n)|$.

Then the summation of these five features is computed by the following formula:

Pre_F $F_{\mathrm{NQ}}(n)=f c 1(n)+f c 2(n)+f c 3(n)+f c 4(n)+f c 5(n)$

Finally the main feature will be computed by:

$F_{N Q}(n)=\left[\begin{array}{cc}\operatorname{Pr} e_{-} F_{N Q}(n), & \text { if } 0 \leq \operatorname{Pr} e_{-} F_{N Q}(n) \leq 1 \\ 1, & \text { if } \operatorname{Pr} e_{-} F_{N Q}(n)>1 \\ 0, & \text { if } \operatorname{Pr} e_{-} F_{N Q}(n)<1\end{array}\right.$

The values greater than $2 \%$ of the maximum of this feature will show the P-wave and T-wave regions in ECG which is marked as pulses. The pulses occur before QRS complexes indicate P-waves and the pulses after QRS shows T-waves as depicted in Figure 2. The details and exact formulation of this procedure can be found in articles. ${ }^{[27]}$

\section{Feature Extraction}

Detecting T-waves from ECG signal prepares the field to extract necessary descriptors from these parts of signal. Totally, 22 features have been considered and extracted from T-wave which consist of two fundamental types of features; There are 17 morphological features and 5 wavelet features. Morphological features include amplitude of T-wave, static and dynamic rising and falling slopes, areas of rising and falling segments, five slopes and five areas respect to split 
falling segment of T-wave into five sections. The other type of features is variances of Daubechies wavelet coefficients which decomposed T-wave segment into five levels. These features have been summarized in Table 1 and shown in Figure 3a-d. As it is evident in Figure 4, the T-wave of three



Figure 2: QRS-Complex and T-Wave separating pulses classes (normal, LQT, and TWA) are very similar together and cannot recognize simply. For this reason, 22 features are selected to classify these types from each other.

\section{Classification}

The classification of diseases is based on Multi Layer Perceptron (MLP) using Artificial Neural Network (ANN) ${ }^{[28,29]}$ with 22 neurons at input, 14 at hidden layer, and one at output which generates 3 integer numbers for 3 classes. All

\begin{tabular}{lll}
\hline \multicolumn{3}{l}{ Table I. Feature description extracted from T-wave } \\
\hline Feature No. & Notation & Description \\
\hline $\mathrm{I}$ & $\mathrm{m}_{\mathrm{sr}}$ & Static rising slope \\
2 & $\mathrm{~m}_{\mathrm{sf}}$ & Static falling slope \\
3 & $\mathrm{~m}_{\mathrm{dr}}$ & Dynamic rising slope \\
4 & $\mathrm{~m}_{\mathrm{df}}$ & Dynamic falling slope \\
5 & $\mathrm{~T}_{\mathrm{p}}$ & Maximum peak of T-wave \\
6 & $\mathrm{~A}_{\mathrm{r}}$ & Rising segment Area \\
7 & $\mathrm{~A}_{\mathrm{f}}$ & Falling segment Area \\
$8-12$ & $\mathrm{~A}_{\mathrm{fl}}, \ldots, \mathrm{A}_{\mathrm{f5}}$ & Falling segment area split into five parts \\
$13-17$ & $\mathrm{~m}_{\mathrm{fl}}, \ldots, \mathrm{m}_{\mathrm{f}}$ & Falling segment slopes split into five parts \\
$18-22$ & $\sigma_{\mathrm{wl}}, \ldots, \sigma_{\mathrm{w} 5}$ & Variance of wavelet coefficients \\
\hline
\end{tabular}

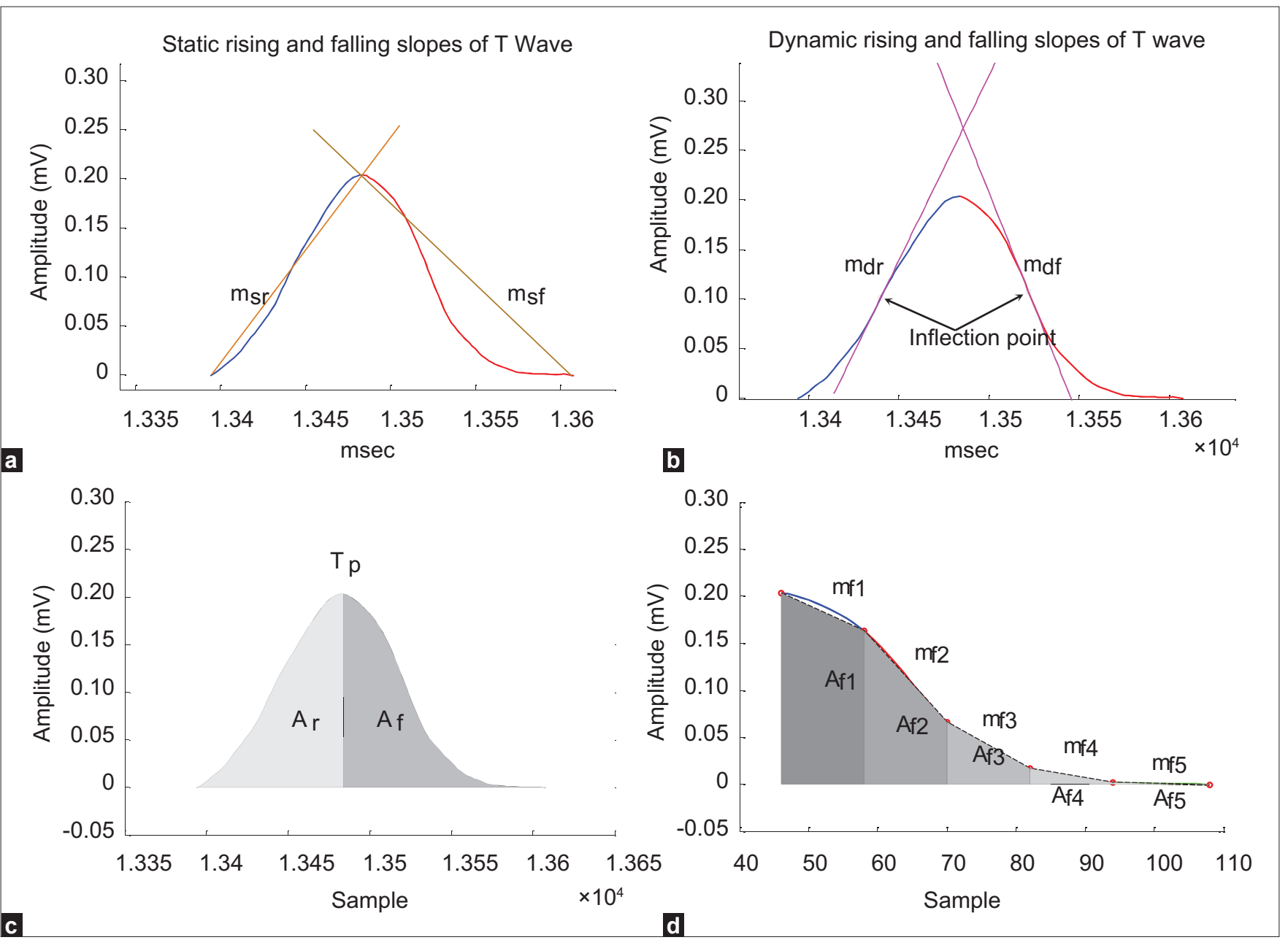

Figure 3: Some morphological features extracted from T-Wave. (a) Dynamic slopes and infection points of T-wave; (b) Static slopes of T-wave; (c) Rising and falling areas of T-wave; (d) Falling area and slopes of T-wave separating to seqments 


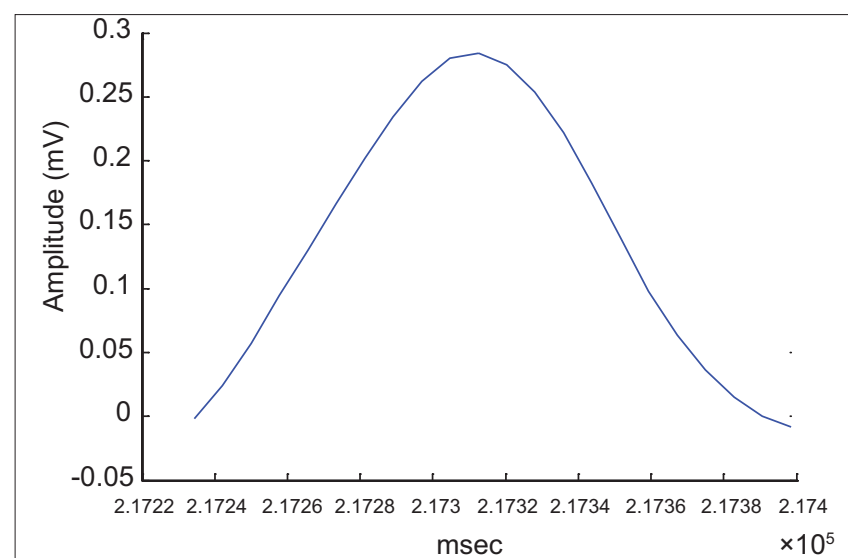

(a)

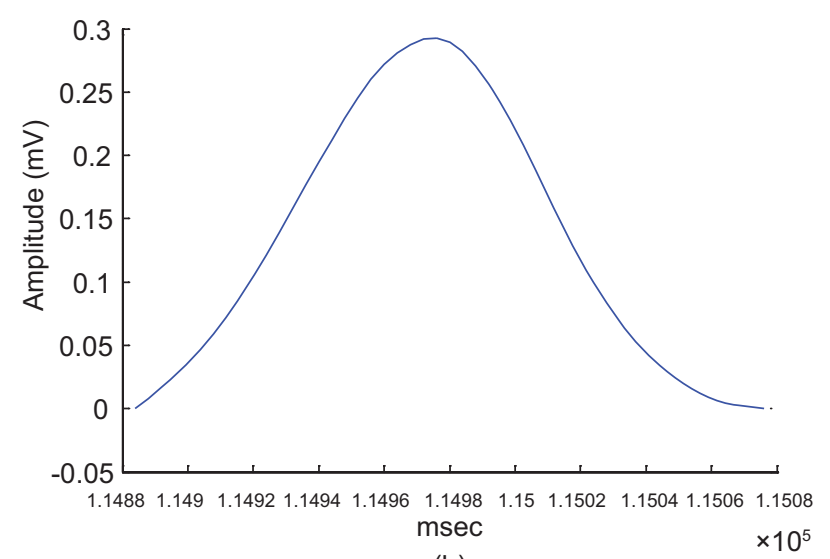

(b)

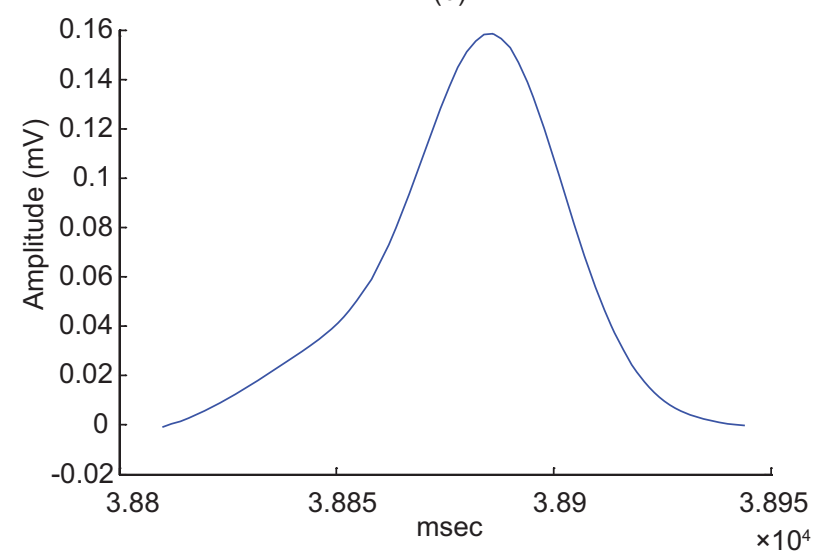

(c)

Figure 4: Typical wave forms fromT-wave section of (a) Normal (b) LQT and (c) TWA

of the 22 features have been scaled and applied to the input of ANN are used and implemented to an appropriate ANN architecture for training and testing as shown in Figure 5. The output of network is determined as normal, LQT, and TWA abnormalities.

\section{RESULTS}

The discussed approach is simulated and applied to normal and abnormal TWA and LQT databases of MIT/BIH arrhythmia

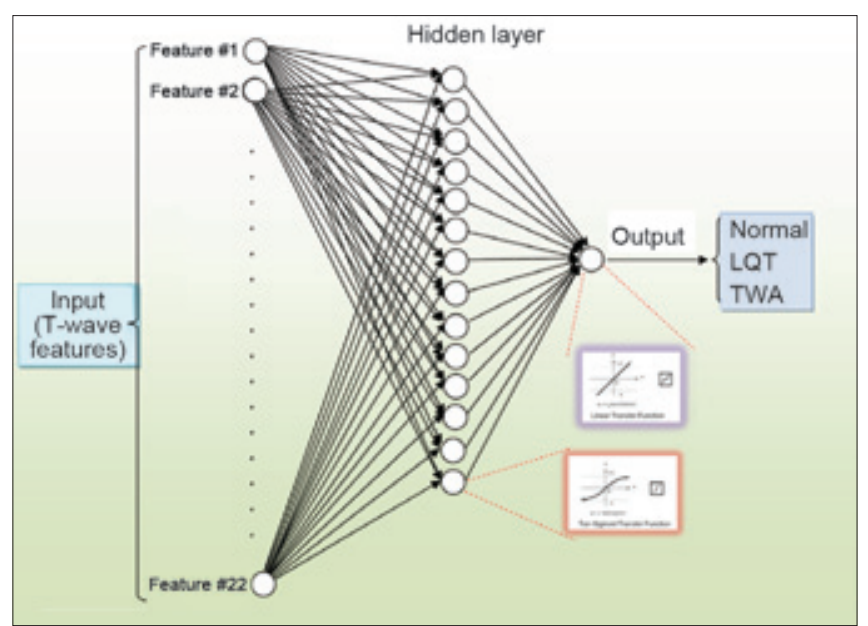

Figure 5: MLP neural network architecture

recordings. The input vectors, which are developed from T-wave features of ECG, are collected and separated into two parts for training and testing the MLP neural network. There are 22 scaled and extracted features containing 17 morphological and 5 wavelet features. Since the T-wave part of an ECG, specially falling interval, illustrates the repolarization phase of heart activity, the information which is accumulated in this section is so significant that it can explain the proper operation of electrical activities in heart. Therefore, these features are rich descriptors for heart performance.

The MIT-BIH database ${ }^{[15-17]}$ has been chosen for implementation of the algorithm in this study. The samples have been taken from three ECG types totally 142 records with the following properties:

- MIT-BIH Normal Sinus Rhythm Database (40 records, $128 \mathrm{~Hz}$ sampling rate);

- $\quad$ The QT Database (47 records, $250 \mathrm{~Hz}$ sampling rate);

- T-Wave Alternans Challenge Database (55 records, $500 \mathrm{~Hz}$ sampling rate).

The learning process to train MLP neural network has been implemented with three different learning sets: 50, 60, and $70 \%$ heartbeats of total recordings. For estimating the performance of discussed approach, three different feature vectors have been developed and tested: Only wavelet features, only morphological features, and both features together. Finally, four performance indices based on ROC (Receiver Operating Characteristics) were computed for normal and abnormal classes: sensitivity $\left(S e_{i}\right)$, specificity $\left(S p_{i}\right)$, positive predictive value $\left(P P V_{i}\right)$ and negative predictive value $\left(N P V_{i}\right)$. They are calculated according to the following relations: ${ }^{[30]}$

$$
\begin{aligned}
& S p_{i}=\frac{T N_{i}}{T N_{i}+F P_{i}}, \quad S e_{i}=\frac{T P_{i}}{T P_{i}+F N_{i}} \\
& N P V_{i}=\frac{T N_{i}}{T N_{i}+F N_{i}}, \quad P P V_{i}=\frac{T P_{i}}{T P_{i}+F P_{i}}
\end{aligned}
$$


where $T P_{i}$ are the number of true positives, $T N_{i}$ are true negatives, $F P_{i}$ are false positives, and $F N_{i}$ are false negatives. The results are representation listed in Tables 2-4 according to different learning sets and descriptor vectors. As it is evident in the results, the network performance differs by changing learning sets and changing the types of features.

The statistical measurements for three features have been depicted in Figure 6 to show the ability of features for the classification of diseases. This describes that the morphological properties, such as falling area and falling slope of T-wave, have different distribution, mean, and variance and can be used for ECG classification.

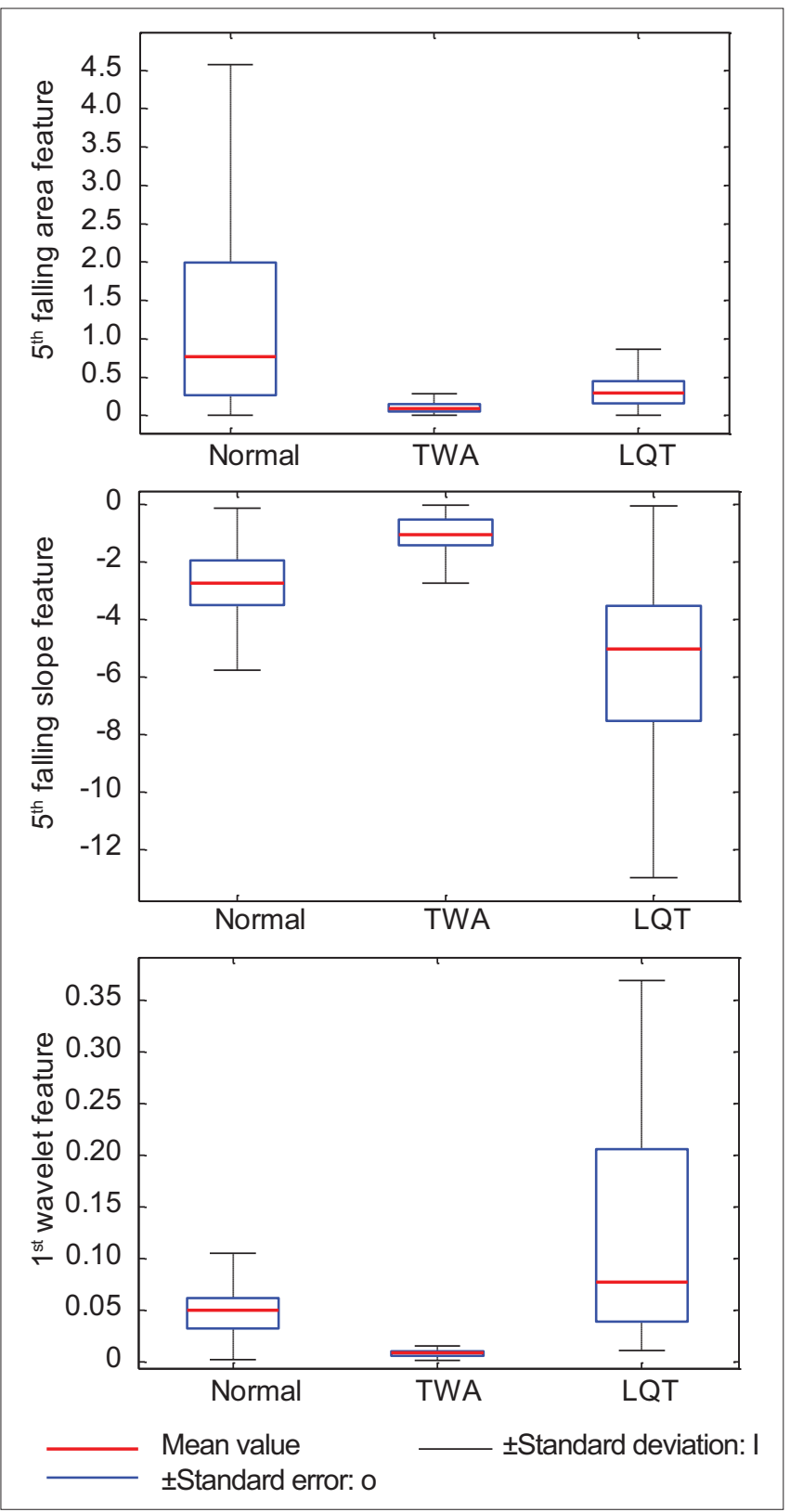

Figure 6: Statistical distribution for three features: $5^{\text {th }}$ falling area, $5^{\text {th }}$ falling slope and $\mathrm{I}^{\text {st }}$ wavelet coef

\section{CONCLUSIONS}

In previous biomedical studies, detecting normal and abnormal beats are considered by applying several methods. These procedures verify the variations of one

\begin{tabular}{|c|c|c|c|}
\hline Normal & $\begin{array}{c}\text { Feature set \# I } \\
5 \text { descriptors } \\
\text { (Wavelets) }\end{array}$ & $\begin{array}{l}\text { Feature set \#2 } \\
\text { I7 descriptors } \\
\text { (Morphology) }\end{array}$ & $\begin{array}{c}\text { Feature set \#3 } \\
22 \text { descriptors } \\
\text { (Total) }\end{array}$ \\
\hline \multicolumn{4}{|c|}{$\begin{array}{l}\text { Learning set \# I } \\
\text { (50\% of data) }\end{array}$} \\
\hline \multicolumn{4}{|l|}{ Index } \\
\hline Spt & 95.01 & 99.43 & 99.8 \\
\hline $\mathrm{Se} \ddagger$ & 90.45 & 98.64 & 99.12 \\
\hline NPV§ & 91.61 & 98.77 & 99.20 \\
\hline PPV* & 94.29 & 99.37 & 99.78 \\
\hline \multicolumn{4}{|c|}{$\begin{array}{l}\text { Learning set \#2 } \\
\text { ( } 60 \% \text { of data) }\end{array}$} \\
\hline \multicolumn{4}{|c|}{ Index } \\
\hline Sp & 96.52 & 99.77 & 99.79 \\
\hline $\mathrm{Se}$ & 90.54 & 98.34 & 99.18 \\
\hline NPV & 91.80 & 98.51 & 99.25 \\
\hline PPV & 95.96 & 99.74 & 99.78 \\
\hline \multicolumn{4}{|c|}{$\begin{array}{l}\text { Learning set \#3 } \\
\text { ( } 70 \% \text { of data) }\end{array}$} \\
\hline \multicolumn{4}{|l|}{ Index } \\
\hline Sp & 96.77 & 99.66 & 99.89 \\
\hline $\mathrm{Se}$ & 88.50 & 98.97 & 99.09 \\
\hline NPV & 90.16 & 99.06 & 99.17 \\
\hline PPV & 96.18 & 99.63 & 99.88 \\
\hline
\end{tabular}

† Sp - Specificity; ‡ Se - Sensitivity; § NPV - Negative predictive value; * PPV - Positive predictive value; Figures are in percentage

\begin{tabular}{|c|c|c|c|}
\hline LQT & $\begin{array}{c}\text { Feature set \# I } \\
5 \text { descriptors } \\
\text { (Wavelets) }\end{array}$ & $\begin{array}{c}\text { Feature set \#2 } \\
17 \text { descriptors } \\
\text { (Morphology) }\end{array}$ & $\begin{array}{c}\text { Feature set \#3 } \\
22 \text { descriptors } \\
\text { (Total) }\end{array}$ \\
\hline \multicolumn{4}{|c|}{$\begin{array}{l}\text { Learning set \# I } \\
\text { (50\% of data) }\end{array}$} \\
\hline \multicolumn{4}{|l|}{ Index } \\
\hline Sp & 95.82 & 99.40 & 99.89 \\
\hline $\mathrm{Se}$ & 73.07 & 96.51 & 98.45 \\
\hline NPV & 88.74 & 98.44 & 99.30 \\
\hline PPV & 88.78 & 98.65 & 99.76 \\
\hline \multicolumn{4}{|c|}{$\begin{array}{l}\text { Learning set \#2 } \\
\text { (60\% of data) }\end{array}$} \\
\hline \multicolumn{4}{|c|}{ Index } \\
\hline Sp & 93.93 & 99.46 & 99.90 \\
\hline $\mathrm{Se}$ & 70.47 & 96.35 & 99.24 \\
\hline NPV & 87.55 & 98.36 & 99.65 \\
\hline PPV & 84.02 & 98.78 & 99.78 \\
\hline \multicolumn{4}{|c|}{$\begin{array}{l}\text { Learning set \#3 } \\
\text { (70\% of data) }\end{array}$} \\
\hline \multicolumn{4}{|l|}{ Index } \\
\hline Sp & 95.25 & 99.32 & 99.89 \\
\hline $\mathrm{Se}$ & 71.77 & 98.75 & 99.71 \\
\hline NPV & 88.10 & 99.43 & 99.96 \\
\hline PPV & 87.33 & 98.52 & 99.76 \\
\hline
\end{tabular}

† Sp - Specificity; ¥ Se - Sensitivity; § NPV - Negative predictive value; * PPV - Positive predictive value; Figures are in percentage 


\begin{tabular}{|c|c|c|c|}
\hline TWA & $\begin{array}{c}\text { Feature set \# I } \\
5 \text { descriptors } \\
\text { (Wavelets) }\end{array}$ & $\begin{array}{c}\text { Feature set \#2 } \\
17 \text { descriptors } \\
\text { (Morphology) }\end{array}$ & $\begin{array}{c}\text { Feature set \#3 } \\
22 \text { descriptors } \\
\text { (Total) }\end{array}$ \\
\hline \multicolumn{4}{|c|}{$\begin{array}{l}\text { Learning set \# I } \\
\text { (50\% of data) }\end{array}$} \\
\hline \multicolumn{4}{|l|}{ Index } \\
\hline Sp & 86.95 & 97.98 & 98.96 \\
\hline $\mathrm{Se}$ & 86.64 & 97.34 & 99.56 \\
\hline NPV & 96.02 & 99.27 & 96.27 \\
\hline PPV & 64.13 & 92.87 & 99.88 \\
\hline \multicolumn{4}{|c|}{$\begin{array}{l}\text { Learning set \#2 } \\
\text { (60\% of data) }\end{array}$} \\
\hline \multicolumn{4}{|l|}{ Index } \\
\hline Sp & 85.15 & 97.72 & 99.25 \\
\hline $\mathrm{Se}$ & 81.11 & 98.30 & 99.37 \\
\hline NPV & 94.38 & 99.53 & 99.83 \\
\hline PPV & 59.44 & 92.05 & 97.25 \\
\hline \multicolumn{4}{|c|}{$\begin{array}{l}\text { Learning set \#3 } \\
\text { ( } 70 \% \text { of data) }\end{array}$} \\
\hline \multicolumn{4}{|l|}{ Index } \\
\hline Sp & 84.10 & 99.03 & 99.43 \\
\hline $\mathrm{Se}$ & 84.69 & 97.49 & 99.73 \\
\hline NPV & 95.43 & 99.33 & 99.93 \\
\hline PPV & 58.34 & 96.35 & 97.84 \\
\hline
\end{tabular}

† Sp - Specificity; ¥ Se - Sensitivity; § NPV - Negative predictive value;

* PPV - Positive predictive value; Figures are in percentage

beat against others to find out abnormal beats. Regarding the new suggestions of cardiologists, some diseases, such as LQT and TWA, have imperceptible effect on time and amplitude of T-wave interval. In contrary to previous articles, in this work, disease detection (LQT syndrome and TWA) has been performed using apparently normal beats.

In some researches, several algorithms have been developed for T-wave detection. The accuracy reported in these articles is satisfactory. ${ }^{[24-26,28,29,31]}$ In this study, the T-wave detection based on threshold method developed in ${ }^{[28]}$ with $96.98 \%$ accuracy is used. This performance is more accurate compared with other methods.

According to the achieved results, it is obvious that T-wave features in sinus ECG signals have the capability to separate these diseases. Since the falling slope of the T-wave is associated with the repolarization phase of heart activity and preparing of heart muscles for next oscillation, this section contains significant morphological descriptors and has the necessary information to classify heartbeats. The specificity of mentioned approach depends on the quantity of learning set and feature types for neural network training. The morphological features of T-wave have also more effect on the classification performance in LQT, TWA, and normal samples compared with wavelet features.

\section{ACKNOWLEDGMENT}

This study was supported by Isfahan University of Medical Sciences and Shiraz Kowsar Heart Clinic, with special thanks to Dr. M.H. Nikoo, Dr. P. Setoodeh, Ms. N. Karami and Mr. A. Fakhrpour.

\section{REFERENCES}

1. Takagi M, Yoshikawa JT. Wave alternans and ventricular tachyarrhythmia risk stratification: A Review. Indian Pacing Electrophysiol J 2003;3: 67-73.

2. Bernardo D, Murray A. Explaining the T-wave shape in the ECG. Nature 2000;403:40.

3. Jain VK. ECG waveform feature extraction and its application to automated prognosis. Int J Parallel Programming 1973;2:231-47.

4. Lin KP, Chang WH. QRS Feature Extraction Using Linear Prediction. IEEE Trans Biomed Eng 1989;36:1050-5.

5. Osowski S, Linh TH. ECG beat recognition using fuzzy hybrid neural network. IEEE Trans Biomed Eng 2001;48:1265-71.

6. Engin M. ECG beat classification using neuro-fuzzy network. Pattern Recognit Lett 2004;25:1715-22.

7. Jekova I, Bortolan G, Christov I. Assessment and comparison of different methods for heartbeat classification. Med Eng Phys 2008;30: 248-57.

8. Asl B, Setarehdan SK, Mohebbi M. Support vector machine-based arrhythmia classification using reduced features of heart rate variability signal. Artif Intell Med 2008;44:51-64.

9. Vaglio M, Couderc JP, McNitt S, Xia X, Moss AJ, Zareba W. A quantitative assessment of T-wave morphology in LQT1, LQT2, and healthy individuals based on Holter recording technology. Heart Rhythm 2008;5:11-8.

10. Couderc JP, McNitt S, Xia J, Zareba W, Moss AJ. Repolarization morphology in adult LQT2 carriers with borderline prolonged QTC interval. Heart Rhythm 2006;3:1460-6.

11. Boix M, Cantó B, Cuesta D, Micó P. Using the Wavelet Transform for T-wave alternans detection. Math Comput Model 2009;50:738-42.

12. Romero I, Grubb NR, Clegg GR, Robertson CE, Addison PS, Watson JN. T-wave alternans found in preventricular tachyarrhythmias in CCU patients using a wavelet transform-based methodology. IEEE Trans Biomed Eng 2008;55:2658-65.

13. Jie Zhao, Jie Tian, Yan-Na Zhao, Ji-Kui Wang, Jian-Gong Xu, Fang-Zhou $\mathrm{Xu}$. Detection of T-wave alternans based on the maximum of T waves. 3rd International Conference on Bioinformatics and Biomedical Engineering (ICBBE): Beijing; 2009. p. 1-4.

14. Ghaffari A, Homaeinezhad MR, Atarod M, Rahmani R. Detecting and quantifying T-wave alternans using the correlation method and comparison with the FFT-based method. Conference on Computers in Cardiology: Bologna; 2008. p. 761-4.

15. The MIT-BIH Normal Sinus Rhythm Database. Available from: http:// www.physionet.org/physiobank/database/nsrdb/ [Last accessed on 2010 Jun 01].

16. Laguna P, Mark R, Golberger A, Moody GB. A database for evaluation of algorithms for measurement of QT and other waveform intervals in the ECG. Comp Card 1997;24:673-6. Available from: http:/www. physionet.org/physiobank/database/qtdb/ [Last accessed on $2011 \mathrm{Sept}$ 26].

17. T-Wave Alternans Challenge Database. Available from: http://www. physionet.org/physiobank/database/twadb/, [Last accessed on 2010 Jun 01].

18. Friesen GM, Jannett TC, Jadallah MA, Yates SL, Quint SR, Nagle HT. A comparison of the noise sensitivity of nine QRS detection algorithms. IEEE Trans Biomed Eng 1990;37:85-98.

19 RM Rangayyan. Biomedical Signal Analysis: A Case Study Apprach. John Wiley \& Sons, Inc., 2002.

20. Rangayyan RM. Biomedical signal analysis: A case study apprach. New York: JohnWiley and Sons, Inc.; 2002. 
21. Chouhan VS, Mehta SS. Total removal of baseline drift from ECG signal, in Proceedings of International Conference on Computing: Theory and Applications: Kolkata; 2007. p. 512-5.

22. Zeraatkar E, Kermani S, Mehridehnavi AR, Aminzadeh A. Improving QRS detection for artifacts reduction. ICBME2010: 17th Iranian Conference on Biomedical Engineering: Isfahan; 2010. p. 1-4.

23. ChouhanVS, Mehta SS. Detection of QRS complexes in 12-lead ECG using adaptive quantized threshold. Int J Computer Sci Netw Secur 2008;8:155-63.

24. Krimi S, Ouni K, Ellouze N. T-Wave detection based on an adjusted wavelet transform modulus maxima. Int J Biol Sci 2006;1:128-32.

25. Li NQ, Wang ZS. T-Wave detection in electrocardiogram signal based on independent sub-band function. Intelligent Syst Appl 2009;2:1-3.

26. Tan KF, Chan KL, Choi K. Detection of the QRS-complex, P-wave, and T-wave in electrocardiogram. Proceedings of the First International Conference on Advances in Medical Signal Processing and Information Processing, IEE conference publication, No. 476. 2000. p. 41-7.

27. Daskalov IK, Christov $2^{\text {nd }}$. Automatic detection of the electrocardiogram T-wave end. Med Biol Eng Comput 1999;37:348-53.
28. ChouhanVS, Mehta SS. Threshold-based Detection of P and T-wave in ECG using New Feature Signal. (IJCSNS) Int J Computer Sci Netw Secur 2008;8:144-53.

29. Haykin S. Neural Networks: A Comprehensive Foundation. New York: McMillan; 1994.

30. Mehridehnavi AR. Classification of the different cancerous animal tissues on the basis of their 1H NMR spectra using different types of Artificial Neural Networks. RPS Res Pharm Sci 2007;2:53-9.

31. Metz CE. Basic Principles of ROC Analysis. Semin Nucl Med 1978;8:283-98.

32. Goutas A, Ferdi Y, Herbeuval JP. Digital fractional order differentiation based algorithm for $\mathrm{P}$ and T-waves detection and delineation. ITBMRBM 2005;2:127-32.

How to cite this article: Zeraatkar E, Kermani S, Mehridehnavi A, Aminzadeh A, Zeraatkar E, Sanei H. Arrhythmia Detection based on morphological and time-frequency features of T-wave in electrocardiogram. J Med Sign Sens 2011;2:99-106

Source of Support: Nil, Conflict of Interest: None declared

\section{BIOGRAPHIES}



Elham Zeraatkar received her B.S. Degree in Electrical Engineering from Shariati Technical University (Iran) in 2004 and her M.Sc. degree in Biomedical Engineering from Departments of Physics and Biomedical Engineering, Isfahan University of Medical Sciences, Isfahan. Her interest is in biomedical signal processing, Fuzzy logic and Neural Network.

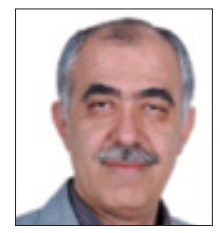

Saeed Kermani obtained his BS from the Department of Electrical Engineering of Isfahan University of Technology in Isfahan, Iran, 1987, and he received the MS in Bioelectric Engineering from Sharif University of Technology, in 1992 and his $\mathrm{PhD}$ in Bioelectric Engineering at AmirKabir University of Technology, Tehran, Iran, in 2008. He is Assistant Professor of Medical Engineering at the Department of Medical Physics and Medical Engineering in the School of Medicine of Isfahan University of Medical Sciences, Iran. His research interests are in biomedical signal and image processing techniques

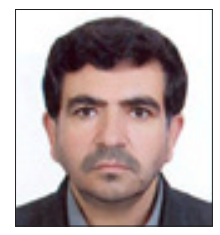

Alireza Mehridehnavi was born in Isfahan province at 1961 . He had educated in Electronic Engineering at Isfahan University of Technology at 1988. He had finished Master of Engineering in Measurement and Instrumentation at Indian Institute of Technology Roorkee (IIT Roorkee) in India at 1992. He has finished his $\mathrm{PhD}$ in Medical Engineering at Liverpool University in UK at 1996. He currently is an Associate
Professor of Medical Engineering at Medical Physics and Engineering Department in Medical School of Isfahan university of Medical Sciences.

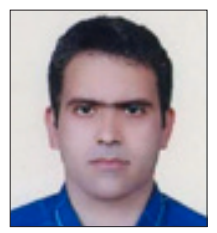

A. Aminzadeh received his B.S. Degree in Electrical Engineering from Shiraz University (Iran) in 2000 and his M.Sc. degree in Control Engineering from Shiraz University in 2003. He has worked in a number of projects related to Real-Time Control, Multiple-Modeling and Heating-Plants. His current research interests include Classification of Biomedical Signals, Neural Networks, Electronic Control Unit of Internal Combustion Engines and Navigation Systems.

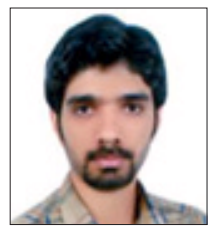

E. Zeraatkar received his B.S. Degree in Control Engineering from Shiraz University (Iran) in 2008 and his M.Sc. degree in Control Engineering from Shiraz University in 2012. His interest is in optimizing convergence in numerical methods such as Neural Networks. Recently he is focused on biomedical signal processing and classification methods for such signals.

Hamid Saneei obtained his MD in 1987, then graduated as a specialist in internal medicine and got his fellow in cardiovascular disease respectively in 1990 and 1996 Isfahan University of Medical Sciences, Iran. He is Associate Professor of cardiology and head of internal medicine department in the School of Medicine of Isfahan University of Medical Sciences, Iran. His research interests are cardiac signal, image and interventional cardiology. 